\title{
Polymicrobial Bacteremia With Actinomyces odontolyti- cus, Fusobacterium and Atopobium parvulum Secondary to Septic Thrombophlebitis in an Immunocompetent Patient
}

\author{
Cassandra Townsend ${ }^{\mathrm{a}}$, Sandra Wong ${ }^{\mathrm{b}}$, Linda Wang ${ }^{\mathrm{a}}$, Raymond Kao ${ }^{\mathrm{c}, \mathrm{d}}$
}

\begin{abstract}
A 25-year-old immunocompetent man was found to have polymicrobial bacteremia secondary to a right arm septic thrombus. His blood cultures grew Actinomyces odontolyticus, Fusobacterium and Atopobium parvulum. Co-infections with these three bacteria are rarely documented and this is the first known reported case of thrombophlebitis associated with these bacteria.
\end{abstract}

Keywords: Actinomyces odontolyticus; Fusobacterium; Atopobium parvulum; Septic thrombophlebitis; Immunocompetent

\section{Introduction}

Actinomyces is an anaerobic gram-positive bacteria typically residing in the oropharynx and intestinal tract as commensal flora. More than 30 species have been described, Actinomyces israelii being the most prevalent disease-causing species [1]. Colonization is ubiquitous in healthy individuals, but poor oral hygiene, immunosuppression, and disruption of local tissue predispose to suppurative infections [2].

Fusobacterium is a gram-negative anaerobe that is most often found in oral flora. This pathogen is most commonly seen in clinically significant anaerobic infections, namely abscesses in the head and neck, endocarditis, bacteremia and soft tissue infections [3]. Fusobacterium necrophorum is one of the most virulent pathogens in the class and a well-documented cause of Lemierre's syndrome [4].

Atopobium parvulum is a gram-positive obligate anaerobe

Manuscript accepted for publication October 26, 2016

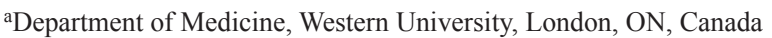
bepartment of Pharmacy, London Health Science Centre, London, ON, Canada

'Division of Critical Care, London Health Sciences Centre, London, ON, Canada

${ }^{\mathrm{d} C}$ Corresponding Author: Raymond Kao, Division of Critical Care, London Health Sciences Centre, 800 Commissioners Road East, London, ON N6A 5W9, Canada. Email: Raymond.Kao@lhsc.on.ca

doi: http://dx.doi.org/10.14740/jmc2685w that is frequently isolated from the oral cavity. It has been isolated on the dorsum of the tongue in patients with halitosis and has also been seen to cause infections of the oral cavity [5].

We present a case of an immunocompetent young man with septic thrombosis in his right arm and Actinomyces odontolyticus, Fusobacterium and Atopobium parvulum bacteremia.

\section{Case Report}

A 25-year-old male with a complex orthopedic history presented to the emergency department complaining of acute onset right shoulder pain, associated with swelling and erythema. With regard to his orthopedic history, it is significant for a nonunion fracture of the tibia after an all-terrain vehicle rollover. This was initially repaired by an open reduction and internal fixation. After the fracture failed to heal, an intermedullary nail and bone graft were used for stabilization. The hardware was removed after osteomyelitis at the fracture site of the right tibia developed. Wound cultures were positive for methicillinsensitive Staphylococcus aureus (MSSA) and Enterococcus faecalis. After irrigation and debridement of the wound site, a 6-week course of intravenous (IV) piperacillin-tazobactam 4.5 g every $8 \mathrm{~h}$ via a right arm peripherally inserted central catheter (PICC) was administered. The PICC was not removed after completion of the antibiotic and the patient removed it himself.

On examination, his vital signs were remarkable for a temperature of $38.6^{\circ} \mathrm{C}$ and sinus tachycardia of 115 beats per minute. His oral hygiene was poor. He denied any history of intravenous drug abuse. His hygienic practices surrounding previous PICC care were unknown. The initial blood work revealed white blood cell count of $21.7 \times 10^{9} / \mathrm{L}$, with a neutrophilia of $18.7 \times 10^{9} / \mathrm{L}$. The venous blood gas showed a respiratory alkalosis, $\mathrm{pH} 7.53, \mathrm{pCO}_{2} 32 \mathrm{~mm} \mathrm{Hg}$, and $\mathrm{HCO}_{3} 27$ $\mathrm{mEq} / \mathrm{L}$. His nasal-pharyngeal screen for methicillin-resistant Staphylococcus aureus (MRSA) was negative. HIV and hepatitis screens were negative.

We treated our patient empirically with piperacillin-tazobactam $4.5 \mathrm{~g}$ IV every $8 \mathrm{~h}$ and vancomycin $1 \mathrm{~g}$ IV every 24 $\mathrm{h}$. Ultrasound of the right arm veins demonstrated a non-occlusive thrombus in the right basilic vein, extending through the axillary and subclavian veins into the right brachiocephalic vein. He was started on rivaroxaban for therapeutic anticoagu- 
lation. Preliminary blood cultures at $24 \mathrm{~h}$ were negative and antibiotics were stopped. Upon discharge, he was apyrexic and given a follow-up appointment with the hematology service. At $72 \mathrm{~h}$ from his initial blood collection, preliminary cultures were positive for Actinomyces odontolyticus and Fusobacterium. The hospital staff was unsuccessful in contacting the patient for re-admission to the hospital.

Five days after discharge, the patient presented to the emergency room complaining of persistent malaise, fever, chills, nausea, vomiting and pain in his right shoulder and axilla. On examination, he was afebrile, and tachycardic at 110 beats per minute, all other vitals were unremarkable. He appeared generally unwell and was tender to palpation in the right shoulder and axilla. Based on the previous positive cultures, he was started on piperacillin-tazobactam $4.5 \mathrm{~g}$ IV every $6 \mathrm{~h}$ and resuscitated with intravenous fluids. The initial laboratory results revealed hemoglobin of $129 \mathrm{~g} / \mathrm{L}$, leukocytosis of $20.1 \times 10^{9} / \mathrm{L}$, neutrophilia at $17.8 \times 10^{9} / \mathrm{L}$ and C-reactive protein (CRP) of $176.1 \mathrm{mg} / \mathrm{L}$. The computed tomography $(\mathrm{CT})$ scan of the thorax was performed to rule out necrotizing fasciitis and assess for thrombus extension. CT revealed that the thrombus had progressed into the right innominate vein from the right brachiocephalic vein. There was no comment of extension into the internal jugular vein. The hematology service suggested that thrombus extension was not a failure of anticoagulation treatment and that the rivaroxaban regimen should be continued. Blood cultures were repeated and found to be positive again for Actinomyces odontolyticus in two sets and Fusobacterium in one set. After treatment with IV antibiotics for 6 days, another set of blood cultures were subsequently drawn and they remained positive for Actinomyces odontolyticus in two cultures and Atopobium parvulum was found in one culture. The Actinomyces was sensitive to clindamycin, meropenem, cefotaxime, and piperacillin-tazobactam and resistant to metronidazole. Both Fusobacterium and Atopobium parvulum were routinely susceptible to penicillin.

The infectious disease team was consulted and recommended to continue 6 weeks of piperacillin-tazobactam 4.5 g IV every $6 \mathrm{~h}$, followed by amoxicillin-clavulanic acid 875 mg orally every $12 \mathrm{~h}$ for at least 6 months thereafter. A transthoracic echocardiogram (TTE) was negative for infective endocarditis. The patient was discharged from the hospital with a normal CRP, negative repeat blood cultures and outpatient follow-up.

\section{Discussion}

A variety of Actinomyces odontolyticus culture-positive infections have been documented in the literature; however, the incidence of Actinomyces odontolyticus bacteremia is less common $[6,7]$. It has been documented in patients who are clearly immunosuppressed $[6,7]$ or have a significant risk factor for infection, including intravenous drug use [8] and previous dental manipulation [9]. Many reported cases of Actinomyces odontolyticus infection were noted to have concomitant growth of another bacteria, which raises the possibility that a co-infection enables it to be pathogenic [10]. Furthermore, few reports of $A$. odontolyticus bacteremia caused by a septic thrombus have been reported. Previous case reports include a recurrent thrombus in the femoral vein in a young man who was an intravenous drug user [8].

We report an immunocompetent individual, who had several risk factors for bacteremia including an indwelling catheter, poor oral hygiene and chronic osteomyelitis. Recent case reports suggest that patients with post-traumatic osteomyelitis were found to have a decreased number of CD5, CD4, CD8 and natural killer cells, most likely secondary to a defect in cytokine production that is presumably induced by trauma [11]. Additionally, the phagocytic capability of immune cells [12] and overall immunologic function is compromised [11, 12].

Our patient repeatedly denied any improper management of the PICC line and intravenous drug abuse. The patient refused radiological imaging of his lower limb, so we were unable to rule out additional pathology. We recognize that this patient could have had defects in his immune system from his chronic osteomyelitis $[11,12]$, that when coupled with poor oral hygiene could have predisposed him to an unusual polymicrobial bacteremia.

Actinomyces odontolyticus and co-infection with Fusobacterium bacteremia have been documented; however, coinfection with Atopobium parvulum has not been reported. We question whether this is due to recent technological advancements in clinical microbiology. The presence of this oral flora is more easily identified using DNA detection methods. While this method is often not practical within the demands of a clinical microbiology laboratory, it has highlighted the need for more precise technology. Our institution uses matrix-assisted laser desorption/ionization (MALDI-TOF) mass spectrometry, which allows for some of these previously missed organisms to be identified and treated [13].

Treatment of a polymicrobial bacteremia involving these microbes is exceedingly challenging and evidence for mode and duration of therapy is minimal. Nevertheless, high doses of antibiotics are recommended to facilitate adequate drug penetration and prolonged courses of therapy have been suggested to achieve resolution and prevent relapse [14]. Treatment typically involves 4 - 6 weeks of IV antibiotic followed by 6 - 12 months of an oral regimen [6].

According to recent antibiotic resistance data, the drug of choice for Actinomyces odontolyticus is penicillin based therapy as no penicillin resistant isolates were found. Fusobacterium spp. however, shows $12.1 \%$ resistance to penicillin, but none of the isolates were resistant to piperacillin-tazobactam, meropenem or metronidazole. Atopobium spp. was also found to have no resistance to penicillin [15]. Piperacillin-tazobactam and amoxicillin-clavulanic acid for oral step-down was selected for our patient to target all three organisms in the coinfection. Susceptibility testing indicated that this was an appropriate treatment.

\section{Conclusion}

To our knowledge, this is the second case of Actinomyces odontolyticus and Fusobacterium co-infection and the first case of Actinomyces odontolyticus and Atopobium parvulum coinfection (reveal search: Pubmed, Embase search). In patients 
with Actinomyces odontolyticus bacteremia secondary to a septic thrombus, we suggest a prolonged treatment regimen with penicillin-based therapy. These patients should be closely monitored until cultures are sterile. More research is needed to determine guidelines for treating these resilient microbes.

\section{Acknowledgments}

We would like to thank the Infectious Disease and Hematology Services for their assistance with management of this complex case.

\section{Funding}

None.

\section{Conflicts of Interest}

The authors declare that there are no conflicts of interest regarding the publication of this paper.

\section{References}

1. Valour F, Senechal A, Dupieux C, Karsenty J, Lustig S, Breton P, Gleizal A, et al. Actinomycosis: etiology, clinical features, diagnosis, treatment, and management. Infect Drug Resist. 2014;7:183-197.

2. Wong VK, Turmezei TD, Weston VC. Actinomycosis. BMJ. 2011;343:d6099.

3. Lee MJ, Ha YE, Park HY, Lee JH, Lee YJ, Sung KS, Kang CI, et al. Osteomyelitis of a long bone due to Fusobacterium nucleatum and Actinomyces meyeri in an immunocompetent adult: a case report and literature review. BMC Infect Dis. 2012;12:161.

4. Karkos PD, Asrani S, Karkos CD, Leong SC, Theochari EG, Alexopoulou TD, Assimakopoulos AD. Lemierre's syndrome: A systematic review. Laryngoscope. 2009;119(8):1552-1559.
5. Copeland A, Sikorski J, Lapidus A, Nolan M, Del Rio TG, Lucas S, Chen F, et al. Complete genome sequence of Atopobium parvulum type strain (IPP 1246). Stand Genomic Sci. 2009;1(2):166-173.

6. Bassiri AG, Girgis RE, Theodore J. Actinomyces odontolyticus thoracopulmonary infections. Two cases in lung and heart-lung transplant recipients and a review of the literature. Chest. 1996;109(4):1109-1111.

7. Cone LA, Leung MM, Hirschberg J. Actinomyces odontolyticus bacteremia. Emerg Infect Dis. 2003;9(12):16291632.

8. Weiand D, Barlow G. The rising tide of bloodstream infections with Actinomyces species: bimicrobial infection with Actinomyces odontolyticus and Escherichia coli in an intravenous drug user. Oxf Med Case Reports. 2014;2014(9):156-158.

9. Chao CT, Liao CH, Lai CC, Hsueh PR. Liver abscess due to Actinomyces odontolyticus in an immunocompetent patient. Infection. 2011;39(1):77-79.

10. Raoult D, Kohler JL, Gallais H, Estrangin E, Peloux Y, Casanova P. [Fusobacterium necrophorum associated with Actinomyces odontolyticus septicemia ]. Pathol Biol (Paris). 1982;30(6 Pt 2):576-580.

11. Sistermann R, Mollenhoff G, Walz M, Josten C, Muhr G. [A cellular quantitative immune deficiency in chronic post-traumatic osteomyelitis]. Unfallchirurg. 1992;95(5):254-258.

12. Peters KM, Klosterhalfen B, Zwadlo-Klarwasser G, Koberg K, Rosendahl T, Zilkens KW. [Lymphocyte defects in chronic osteomyelitis. A prospective study]. Unfallchirurg. 1993;96(1):29-33.

13. Murray PR. What is new in clinical microbiology-microbial identification by MALDI-TOF mass spectrometry: a paper from the 2011 William Beaumont Hospital Symposium on molecular pathology. J Mol Diagn. 2012;14(5):419-423.

14. Finegold SM, Wexler HM. Present status of therapy for anaerobic infections. Clin Infect Dis. 1996;23:9-14.

15. Marchand-Austin A, Rawte P, Toye B, Jamieson FB, Farrell DJ, Patel SN. Antimicrobial susceptibility of clinical isolates of anaerobic bacteria in Ontario, 2010-2011. Anaerobe. 2014;28:120-125. 